\title{
Updated Clinical Considerations for Dental Implant Therapy in Irradiated Head and Neck Cancer Patients
}

\author{
Takako Imai Tanaka, DDS, FDS RCSEd, ${ }^{1,2}$ Hsun-Liang Chan, DDS, MS, ${ }^{2}$ David Ira Tindle, DDS, MS, ${ }^{2}$ \\ Mark MacEachern, MLIS, ${ }^{3}$ \& Tae-Ju Oh, DDS, MS ${ }^{2}$ \\ ${ }^{1}$ Department of Biomedical \& Diagnostic Sciences, University of Detroit Mercy School of Dentistry, Detroit, MI \\ ${ }^{2}$ Department of Periodontics and Oral Medicine, University of Michigan School of Dentistry, Ann Arbor, Ml \\ ${ }^{3}$ A. Alfred Taubman Health Sciences Library, University of Michigan, Ann Arbor, MI
}

\begin{abstract}
The article is associated with the American College of Prosthodontists' journal-based continuing education program. It is accompanied by an online continuing education activity worth 1 credit. Please visit www.wileyonlinelearning.com/jopr to complete the activity and earn credit.
\end{abstract}

\section{Keywords}

Dental implant; head and neck cancer; irradiation; survival rate; oral rehabilitation.

\section{Correspondence}

Takako I. Tanaka, 2700 MLK Jr. Blvd., Detroit, MI 48208-2576. E-mail:

tanakata@udmercy.edu

The authors deny any conflicts of interest

Accepted November 11, 2012

doi: $10.1111 / j o p r .12028$

\begin{abstract}
An increasing number of reports indicate successful use of dental implants (DI) during oral rehabilitation for head and neck cancer patients undergoing tumor surgery and radiation therapy. Implant-supported dentures are a viable option when patients cannot use conventional dentures due to adverse effects of radiation therapy, including oral dryness or fragile mucosa, in addition to compromised anatomy; however, negative effects of radiation, including osteoradionecrosis, are well documented in the literature, and early loss of implants in irradiated bone has been reported. There is currently no consensus concerning DI safety or clinical guidelines for their use in irradiated head and neck cancer patients. It is important for health care professionals to be aware of the multidimensional risk factors for these patients when planning oral rehabilitation with DIs, and to provide optimal treatment options and maximize the overall treatment outcome. This paper reviews and updates the impact of radiotherapy on DI survival and discusses clinical considerations for DI therapy in irradiated head and neck cancer patients.
\end{abstract}

Cancer of the oral cavity and pharynx, the largest group of head and neck cancers, is the ninth most common cancer in males in the United States. ${ }^{1}$ Approximately 40,000 people will be newly diagnosed with oral cancer with a 5-year survival rate of $57 \% .^{2}$ Surgery is a well-established treatment and may include radiotherapy (RT) and/or chemotherapy. ${ }^{3}$ Reconstruction of major surgical defects is required for a majority of the cases, followed by rehabilitation of missing teeth and restoring orofacial function.

Use of implants for prosthetic reconstruction has dramatically increased due to advancements in materials science and surgical techniques during the past three decades. ${ }^{4}$ Implantsupported dentures seem to be a viable option, especially when RT's adverse effects, such as oral dryness or fragile mucosa, along with compromised anatomy, hamper the use of conventional removable dentures. ${ }^{5}$

An increased number of reports indicate successful implantsupported prostheses in irradiated cancer patients. ${ }^{6-10}$ However, the negative effects of radiation are well documented, ${ }^{11}$ and several studies in both animals and humans have shown an increased risk of early loss of dental implants (DI) in irradiated bone. ${ }^{12-14}$ There is currently no consensus about the predictability, safety, or clinical guidelines for DI therapy in irradiated head and neck cancer patients. This paper reviews the impact of RT on DI therapy and discusses updated clinical considerations for DI therapy in those patients.

\section{RT and its adverse effects}

Cancer cells are in a continuous state of mitosis. Ionizing radiation produces energy that injures or destroys cells by damaging nuclear DNA or altering the molecular characteristics of individual cells. ${ }^{2}$ Most patients with head and neck cancer receive between 50 and 70 Grays (Gy) as a curative dose. For concomitant use, 45 Gy are used preoperatively and 55 to $60 \mathrm{~Gy}$ postoperatively. These doses are typically fractionated over a period of 5 to 7 weeks, once a day, 5 days a week, with a daily dose of approximately $2 \mathrm{~Gy}^{2}$ Normally, each daily treatment lasts about 10 to 15 minutes. Fractionated radiation is used because in general, normal tissue repairs sub-lethal DNA damage better than tumor tissue, especially in the low-dose range.

Adverse effects of RT include mucositis, hyposalivation, loss of taste, radiation caries, trismus, and osteoradionecrosis (ORN) of the jaw. ORN, ischemic necrosis of bone, is one of the most serious complications. ${ }^{11}$ Initial changes in bone caused by irradiation result from direct injury to the remodeling system (osteocytes, osteoblasts, and osteoclasts). In addition, vascular 




Figure 1 CT scan showing the fracture of the right mandible of a patient after tumor surgery, RT, and $\mathrm{HBO}$.

injury precedes hyperemia, followed by endarteritis, thrombosis, and a progressive occlusion and obliteration of small vessels. With time, the bone marrow exhibits marked acellularity and avascularity, with marked fibrosis and fatty degeneration. ${ }^{14}$ ORN occurs in the mandible more often than the maxilla. Although $30 \%$ of cases may be asymptomatic, more patients with ORN present pain, fistula formation, and in more severe cases, spontaneous bone fracture (Fig 1). Recent systematic reviews found the risk of developing ORN in irradiated head and neck cancer patients as low as $2 \%$; however, the risk can be higher after tooth extraction. ${ }^{15,16}$ While peak time of spontaneous ORN was in the first 2 to 3 years after RT, ${ }^{17}$ the risk of trauma-induced ORN might last indefinitely. ${ }^{11}$ The risk and severity of ORN is known to be directly related not only to radiation dose and the volume of irradiated tissue, but also to the dental health of the patients. $^{15}$

\section{RT on implant survival}

Many studies have shown that DI therapy in irradiated patients is not significantly less favorable than in the non-irradiated population (Table 1). These studies were identified via a PubMed search using Medical Subject Headings and keyword phrases for "dental implants," "dental prosthesis," "radiotherapy," "radiation effects," and other variations. The papers were limited to human studies, English language papers, and papers published since 2000. Clinical reports and studies with a sample size less than 10 were excluded. The identified studies reported implant survival rates or success rates in patients with RT ranging from $74.4 \%$ to $98.9 \%$ with the majority reporting survival rates above $84 \%$. Many additional studies also reported high function of implant-supported dentures in irradiated head and neck patients, with relatively high implant survival rates. ${ }^{18-20}$ However, conflicting results exist. The major concerns for DI therapy in irradiated head and neck cancer patients are the potential for delayed healing and the later risk of ORN. ${ }^{21,22}$ In a recent review of the literature evaluating RT effects on both dental and craniofacial implants in animal and human subjects, the relative risk of implant failure in irradiated bone was found to be 2 to 3 times greater than that of non-irradiated subjects. ${ }^{23}$ Animal models have revealed compromised osseointegration of DI due to irradiation, such as impaired osteogenesis, bone strength reduction, and significant fibrosis of the periosteum, to be the common end-stage of tissue injury. ${ }^{13,24,25}$ Some human studies show a lower survival rate of implants placed in irradiated bone compared to non-irradiated controls. ${ }^{1,9,12}$ The differing methodologies among these studies, the varying definitions of implant survival and success, the improvements in implant surface features, and variations in treatment modalities may account for the controversies regarding DI predictability in these patients. ${ }^{26-31}$ Many factors influencing treatment outcome have been suggested (Fig 2). In the following section, we will discuss the impact of RT on DI therapy.

\section{Impact of RT on DI therapy}

\section{Radiation dose and implant location}

DI failures were seldom seen at cumulative doses less than 45 $\mathrm{Gy}^{32}$ and more commonly seen at doses greater than $65 \mathrm{~Gy}^{33}$ ORN risk has been reported to be highest in the extraction of mandibular teeth within the radiation field with doses greater than $60 \mathrm{~Gy}^{7}$ Studies have shown that short survival of implants in irradiated patients was significant with total doses $>50$ to 55 Gy. ${ }^{9,22,33}$ Many agree that DI survival in patients treated with cumulative RT doses lower than 50 Gy can be comparable to that in non-irradiated patients. ${ }^{34-36}$

Implants in the mandible have shown higher survival rates than those in the maxilla. ${ }^{34,37}$ As mirrored by DI practice in the general population, ${ }^{31}$ that is most likely due to high bone density of the mandible providing better initial primary implant stability. Visch et al, in their study with 130 consecutive cancer patients irradiated orally over a period of up to 14 years, found implant location in the maxilla or mandible $(59 \%$ and $85 \%$, respectively) as a dominant factor $(p=0.001)$ among other potential factors influencing implant survival. ${ }^{22}$ Nelson et al reported similar results in the maxilla and mandible $(70 \%$ and $92 \%$, respectively) in their first 5 years of follow-up, though long-term survival rates (after 8 years) were found to be equivo$\mathrm{cal}^{28}$ The region of the mandible anterior to the mental foramen is usually exposed to a lower dose than other sites during RT and seems to have better remodeling capability due to additional vascular supply from the facial artery. ${ }^{38,39}$ It must be noted that the radiation dose per implant site ideally should be known to determine the impact of radiation dose on implant survival. ${ }^{4}$ However, it is often not available in the literature. ${ }^{23,40}$

\section{RT modalities and adjuvant chemotherapy}

Advancements in RT techniques have been developed to preserve function of normal tissue and enhance tumor control. Those include Intensity-Modulated Radiation Therapy (IMRT) 


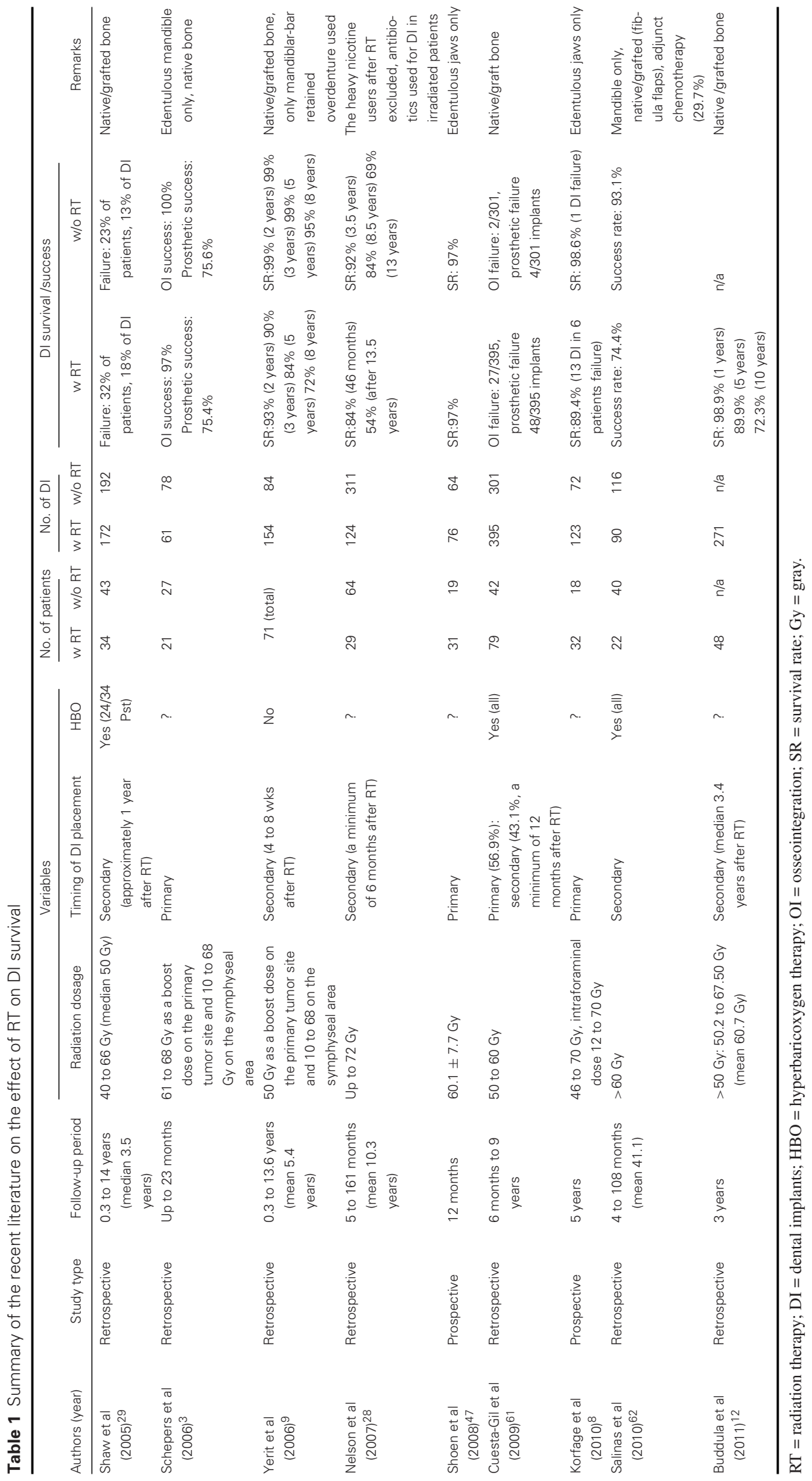




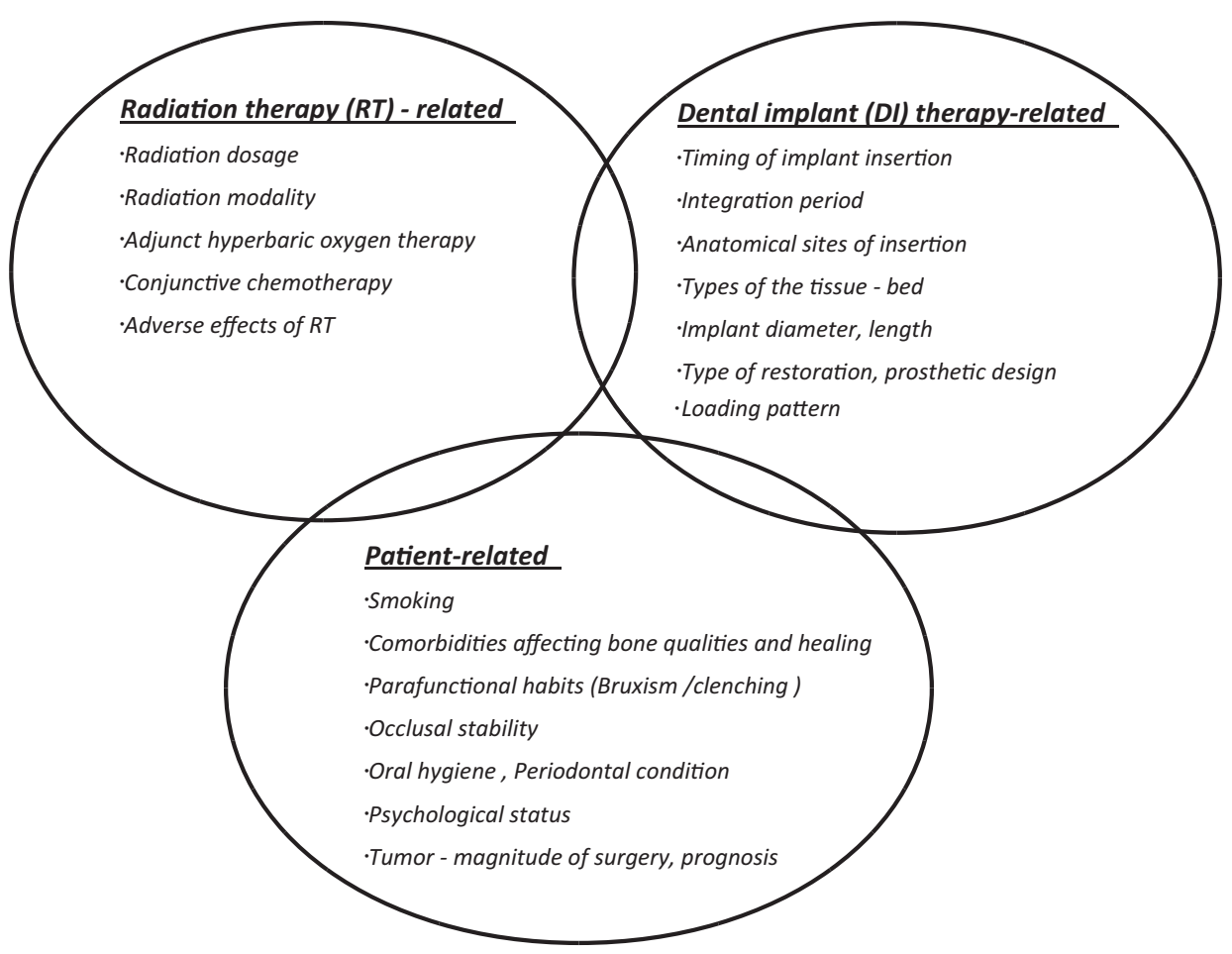

Figure 2 Potential factors impacting dental implant therapy in irradiated head and neck cancer patients.

and altered fractionation schedules (hyperfractionation and accelerated fractionation). IMRT is a computer-driven technology using rapid radiation beams of varying intensity to confine the dose to the target tissues. ${ }^{41}$ IMRT in the head and neck region aims to preserve the parotid glands particularly, resulting in prevention of hyposalivation. Hyposalivation frequently results in dental caries and necessitates tooth extraction, which increases the risk of ORN. ${ }^{16}$ Hyperfractionation delivers an increased number of fractions and total dose with a smaller dose per fraction. Accelerated fractionation provides radiation at a faster rate of accumulation than that of standard fractionation, and with a similar total dose to conventional $\mathrm{RT}^{40}$ A recent systematic review found that risk of ORN after tooth extraction in irradiated patients was reduced by accelerated fractionation with dose reduction but elevated by hyperfractionation. ${ }^{16}$ However, studies focusing on the impact of RT modality on DI survival are scarce at this time.

Concomitant chemotherapy is often incorporated in cancer therapy to augment the anti-tumor effect of RT. Major side effects include acute mucositis and altered taste, which can be multiplied with RT; however, currently there is little evidence that chemotherapy influences DI therapy. ${ }^{42}$ Nabil and Samman did not find significantly increased ORN risk in patients receiving chemoradiotherapy. ${ }^{16}$

\section{Adjunctive use of hyperbaric oxygen therapy (HBO)}

HBO has been used for a wide range of medical conditions such as syphilis, multiple sclerosis, and myocardial infarc- tion. It raises levels and diffusion of oxygen in local tissue by inducing angiogenesis, increasing bone metabolism (enhancing osteoblast repopulation and fibroblast function), and stimulating collagen synthesis. ${ }^{11}$ Therefore, it is expected that $\mathrm{HBO}$ increases the capacity to repair tissue damaged by RT.

The protocol of HBO used after RT in the head and neck region usually includes 20 to 30 sessions (lasting 90 minutes each) prior and 10 minutes after tooth extraction or implant placement, at a compression of 2.4 atmospheres absolute pressure with $100 \%$ oxygen. ${ }^{43}$ Many support adjunctive use of $\mathrm{HBO}$ to prevent and manage ORN, especially when the implant site is irradiated with more than 50 Gy and shows clinical signs of radiation damage. $6,15,35$ Better wound healing on the implant site of the mandible was noted in irradiated patients who received HBO therapy during the 3 to 7 year follow-up period. ${ }^{44}$ Granström et al $^{45}$ found significantly lower failure rates of craniofacial implants including DI in HBO-treated patients than those in the non-HBO-treated group (8.1\% vs. $53.7 \%$, respectively); however, opinions conflict on the prophylactic effect of HBO in reducing the risk of RT-induced ORN, and it has not been universally accepted in dentistry. Studies have shown that HBO could not enhance implant survival in irradiated mandibular bone. ${ }^{29,46}$ Limited accessibility and its high costs in time and money might be concerns. ${ }^{46} \mathrm{HBO}$ requires approximately 1 month before tooth extraction, which may not always be practical for symptomatic cases. Potential complications such as middle ear barotraumas and myopia must also be taken into consideration, as well as contraindications including uncontrolled COPD. ${ }^{46}$ 


\section{Timing of implant placement related to $\mathbf{R T}$}

Optimal timing of DI insertion-related RT has been debated. Immediate implant insertion before RT, at the same time as the ablative tumor surgery, is referred to as primary placement, as opposed to secondary placement after RT. Primary placement of implants aims at achieving osseointegration prior to onset of the damaging effects of RT, and early oral rehabilitation by avoiding additional surgery., 37 Overall cost can be reduced; however, indications may be limited to low-grade tumors. Computer-guided implant placement has recently been introduced to improve identification of the ideal implant location during surgery. ${ }^{48-50}$ Secondary placement of implants is probably more common in dental practice because primary placement is not always available to patients in the hospital setting. Delayed DI placement enables assessment of the postsurgical status of the patients (both functionally and psychologically), and more accurate cancer prognosis. ${ }^{29}$ Patients will be given more time to choose prosthetic treatment options after their recovery from tumor therapy and prior to initiation of an extensive dental procedure. $^{24}$

Recent publications have demonstrated encouraging results in primary placement of DI. ${ }^{3,47}$ A human study shows better implant survival in primary placement than in secondary placement in edentulous mandibles of patients with oral squamous cell carcinoma. ${ }^{3}$ However, a recent systematic review by Colella et al found a similar implant failure rate between the two groups ( $3.2 \%$ vs. $5.4 \%$, respectively). ${ }^{32}$ Choice of implant insertion timing (before RT vs. after RT) likely depends on the surgical team's personal preference. ${ }^{11}$ No randomized controlled studies have been conducted regarding timing of implant therapy and implant success.

How long should we wait for implant placement after RT? The answer remains unclear at this time. Several studies showed that 6 months after RT, DI survival was not affected by placement timing. ${ }^{9,22}$ However, to avoid early complications of tumor therapy, most clinicians agree to wait a minimum of 6 to 12 months. ${ }^{24}$ Others have recommended waiting a little longer (12 to 18 months typically) to allow enough time for bone remodeling and muscle healing. ${ }^{51,52}$ Tumor recurrence or developing a second malignancy in the adjacent region must be another important consideration. ${ }^{34,53}$ The first 12 months after tumor therapy is generally considered to be the high risk period of recurrence. A longitudinal prospective study showed $44 \%$ of cancer patients who underwent mandibular resection had recurrence within 13 months of surgery. ${ }^{34}$

It must be noted that the risk of ORN may persist for years after RT in head and neck cancer patients. ${ }^{4,14}$ Progressive loss of capillaries (therefore loss of tissue perfusion) without evidence of spontaneous revascularization over time was found in a study by Marx and Johnson, using serial biopsy specimens from more than 143 irradiated patient mandibles. ${ }^{14}$ Granström et al reported progressive loss of DI due to failure of osseointegration up to 6 years after the placement. ${ }^{54}$

\section{Oral health and psychological status of patients related to $R T$}

Although the direct impact of oral health status on DI survival is not clear in the literature, local effects of RT on periodontal tissue, and attachment loss particularly, have been reported in several studies. ${ }^{55,56}$ Epstein et al observed tooth loss and progressive periodontal attachment loss in teeth within areas of high-dose radiation. ${ }^{55}$ A study by Marques and Dib showed similar results and explained that inadequate homecare resulting from lack of motivation, RT-induced hyposalivation, and limited vertical opening might contribute to such significant periodontal destruction in irradiated head and neck cancer patients. ${ }^{56}$ Poor oral health is known to increase ORN risk. ${ }^{57,58}$ Katsura et al reported that oral conditions such as periodontal pocket depth $>5 \mathrm{~mm}$, dental plaque score $>40 \%$, and alveolar bone loss $>60 \%$, in the first or second year after RT, as well as smoking history after RT were significantly associated with ORN risk. ${ }^{57}$

Patients undergoing cancer therapies are often weak. RT can be detrimental to the patients' quality of life, compromising speech, swallowing, and sensory and masticatory function, in addition to compromised esthetics caused by major surgery. ${ }^{11,29}$ After tumor therapies, patients often become apprehensive about further extensive dental treatment, which results in unloaded implants. ${ }^{3,24}$ Compared to the high success rate of implant osseointegration, a lower success rate for prostheses has been reported. ${ }^{24}$ In the study by Smolka et al, prosthetic success was reported as $42.9 \%$, while implant success was $92 \%$ in 56 cancer patients who had mandibular free flap reconstruction. ${ }^{59}$ Schepers et al found the ultimate rates of functional implantsupported dentures in the postoperative irradiated group versus surgery-only group were $75.4 \%$ versus $75.6 \%$, respectively, compared to a $97 \%$ success rate of implant osseointegration during the 23-month follow-up. ${ }^{3}$ In their study, $24.5 \%$ of the primary-placed implants never became functional because of cancer-related (tumor recurrence or metastasis) or psychological reasons.

\section{Discussion}

Interpretation of published data needs special caution. Neither a high implant survival rate nor success of osseointegration assures functional DI or success of DI therapy in cancer patients; however, with careful case selection, DI therapy in oral rehabilitation in irradiated head and neck cancer patients can be successfully achieved. Before initiation of DI therapy, a patient's level of tolerance must be assured both physically and psychologically. Reasonable oncologic prognosis should be obtained from the physician. The planned implant site of the anterior mandible and a cumulative radiation dosage lower than $50 \mathrm{~Gy}$ may predict the outcome of DI therapy. If possible, consultation with the radiation oncologist is encouraged to obtain radiation dose distribution. A timespan of 12 months between the last RT and implant insertion seems reasonable from both the oncologic and dental prospective; however, later complications of RT, including ORN, are still not clear. In general, placement of a minimal number of implants is recommended. ${ }^{2}$ It is the authors' opinion that options for primary placement of implants should be discussed prior to tumor surgery to avoid potential damage from RT for implant sites such as ORN. The impacts of adjunctive HBO, chemotherapy, or RT modality on implant survival remain uncertain. 
Throughout the oral rehabilitation, it is important to maintain optimal periodontal health with appropriate management of any adverse effects of RT. General known risk factors of DI failure should be controlled to maximize the treatment outcome. Lack of communication and education among the patient and healthcare professionals have been raised as concerns. ${ }^{2}$ New guidelines for prophylactic dental care prior to the head and neck cancer treatment that could damage the oral tissue in cancer patients are on the horizon. ${ }^{11,60}$

\section{Conclusion}

Multidimensional potential risk factors of DI failure must be considered when planning DI therapy in irradiated head and neck patients. Factors focusing on RT were discussed, and the negative impacts of RT on DI therapy are undeniable. The benefit of using implant-supported dentures over conventional dentures must outweigh the risks. Meticulous treatment planning along with careful preoperative oral examination and good coordination with oncologic specialists cannot be overemphasized. It is also important for the dental profession to keep abreast of the latest available RT technologies. Additional evidence-based clinical guidelines for implant use in head and neck patients undergoing RT are expected.

\section{Acknowledgment}

Dr. Robert Nisker is gratefully acknowledged for his contribution of the CT imaging.

\section{References}

1. American Cancer Aociety: Cancer Treatment and Survivorship. 2012; Available at: http://www.cancer.org/acs/groups/content/\% 40epidemiologysurveilance/documents/document/acspc-027 766.pdf (accessed September 9, 2012)

2. Oral Cancer Foundation: Oral Cancer facts. 2012; Available at: http://www.oralcancerfoundation.org/facts/ (accessed September 9, 2012)

3. Schepers RH, Slagter AP, Kaanders JH, et al: Effect of postoperative radiotherapy on the functional result of implants placed during ablative surgery for oral cancer. Int J Oral Maxillofac Surg 2006;35:803-808

4. Granström G: Osseointegration in irradiated cancer patients: an analysis with respect to implant failures. J Oral Maxillofac Surg 2005;63:579-585

5. Dholam KP, Gurav SV: Dental implants in irradiated jaws: a literature review. J Cancer Res Ther 2012;8(Suppl 1):S85-93

6. Barrowman RA, Wilson PR, Wiesenfeld D: Oral rehabilitation with dental implants after cancer treatment. Aust Dent J 2011;56:160-165

7. Jisander S, Grenthe B, Alberius P: Dental implant survival in the irradiated jaw: a preliminary report. Int J Oral Maxillofac Implants 1997;12:643-648

8. Korfage A, Schoen PJ, Raghoebar GM, et al: Benefits of dental implants installed during ablative tumour surgery in oral cancer patients: a prospective 5-year clinical trial. Clin Oral Implants Res 2010;21:971-979
9. Yerit KC, Posch M, Seemann M, et al: Implant survival in mandibles of irradiated oral cancer patients. Clin Oral Implants Res 2006; 17:337-344

10. Linsen SS, Martini M, Stark H: Long-term results of endosteal implants following radical oral cancer surgery with and without adjuvant radiation therapy. Clin Implant Dent Relat Res 2012;14:250-258

11. Vissink A, Burlage FR, Spijkervet FK, et al: Prevention and treatment of the consequences of head and neck radiotherapy. Crit Rev Oral Biol Med 2003;14:213-225

12. Buddula A, Assad DA, Salinas TJ, et al: Survival of dental implants in native and grafted bone in irradiated head and neck cancer patients: a retrospective analysis. Indian J Dent Res 2011;22:644-648

13. Verdonck HW, Meijer GJ, Laurin T, et al: Implant stability during osseointegration in irradiated and non-irradiated minipig alveolar bone: an experimental study. Clin Oral Implants Res 2008;19:201-206

14. Marx RE, Johnson RP: Studies in the radiobiology of osteoradionecrosis and their clinical significance. Oral Surg Oral Med Oral Pathol 1987;64:379-390

15. Nabil S, Samman N: Risk factors for osteoradionecrosis after head and neck radiation: a systematic review. Oral Surg Oral Med Oral Pathol Oral Radiol 2012;113:54-69

16. Nabil S, Samman N: Incidence and prevention of osteoradionecrosis after dental extraction in irradiated patients: a systematic review. Int J Oral Maxillofac Surg 2011;40:229-243

17. Thorn JJ, Hansen HS, Specht L, et al: Osteoradionecrosis of the jaws: clinical characteristics and relation to the field of irradiation. J Oral Maxillofac Surg 2000;58:1088-1093; discussion 1093-5.

18. Fukuda M, Takahashi T, Nagai H, et al: Implant-supported edentulous maxillary obturators with milled bar attachments after maxillectomy. J Oral Maxillofac Surg 2004;62:799-805

19. Korfage A, Schoen PJ, Raghoebar GM, et al: Five-year follow-up of oral functioning and quality of life in patients with oral cancer with implant-retained mandibular overdentures. Head Neck 2011;33:831-839

20. Taira Y, Sekine J, Sawase T, et al: Implant-retained overdenture following hemiglossectomy: a 10-year clinical case report. J Oral Rehabil 2006;33:313-315

21. Harrison JS, Stratemann S, Redding SW: Dental implants for patients who have had radiation treatment for head and neck cancer. Spec Care Dentist 2003;23:223-229

22. Visch LL, van Waas MA, Schmitz PI, et al: A clinical evaluation of implants in irradiated oral cancer patients. J Dent Res 2002;81:856-859

23. Ihde S, Kopp S, Gundlach K, et al: Effects of radiation therapy on craniofacial and dental implants: a review of the literature. Oral Surg Oral Med Oral Pathol Oral Radiol Endod 2009;107:56-65

24. Asikainen P, Klemetti E, Kotilainen R, et al: Osseointegration of dental implants in bone irradiated with 40, 50 or 60 gy doses. An experimental study with beagle dogs. Clin Oral Implants Res 1998;9:20-25

25. Renou SJ, Guglielmotti MB, de la Torre A, et al: Effect of total body irradiation on peri-implant tissue reaction: an experimental study. Clin Oral Implants Res 2001;12:468-472

26. Carr AB: Oral cancer therapy may influence survival of dental implants. J Evid Based Dent Pract 2011;11:124-126

27. Jokstad A: Can dental implants osseointegrate in oral cancer patients? Evid Based Dent 2011;12:82-83

28. Nelson K, Heberer S, Glatzer C: Survival analysis and clinical evaluation of implant-retained prostheses in oral cancer resection patients over a mean follow-up period of 10 years. J Prosthet Dent 2007;98:405-410 
29. Shaw RJ, Sutton AF, Cawood JI, et al: Oral rehabilitation after treatment for head and neck malignancy. Head Neck 2005;27:459-470

30. Anne-Gaelle B, Samuel S, Julie B, et al: Dental implant placement after mandibular reconstruction by microvascular free fibula flap: current knowledge and remaining questions. Oral Oncol 2011;47:1099-1104

31. Strub JR, Jurdzik BA, Tuna T: Prognosis of immediately loaded implants and their restorations: a systematic literature review. J Oral Rehabil 2012;39:704-717

32. Colella G, Cannavale R, Pentenero M, et al: Oral implants in radiated patients: a systematic review. Int J Oral Maxillofac Implants 2007;22:616-622

33. Granstrom G: Placement of dental implants in irradiated bone: the case for using hyperbaric oxygen. J Oral Maxillofac Surg 2006; $64: 812-818$

34. Garrett N, Roumanas ED, Blackwell KE, et al: Efficacy of conventional and implant-supported mandibular resection prostheses: study overview and treatment outcomes. J Prosthet Dent 2006;96:13-24

35. Larsen PE: Placement of dental implants in the irradiated mandible: a protocol involving adjunctive hyperbaric oxygen. J Oral Maxillofac Surg 1997;55:967-971

36. Sammartino G, Marenzi G, Cioffi I, et al: Implant therapy in irradiated patients. J Craniofac Surg 2011;22:443-445

37. Kovacs AF: Clinical analysis of implant losses in oral tumor and defect patients. Clin Oral Implants Res 2000;11:494-504

38. de Oliveira JA, do Amaral Escada AL, Alves Rezende MC, et al: Analysis of the effects of irradiation in osseointegrated dental implants. Clin Oral Implants Res 2012;23:511-514

39. Nishimura RD, Roumanas E, Beumer J, 3rd, et al: Restoration of irradiated patients using osseointegrated implants: current perspectives. J Prosthet Dent 1998;79:641-647

40. Zackrisson B, Mercke C, Strander H, et al: A systematic overview of radiation therapy effects in head and neck cancer. Acta Oncol 2003;42:443-461

41. Lee N, Puri DR, Blanco AI, et al: Intensity-modulated radiation therapy in head and neck cancers: an update. Head Neck 2007;29:387-400

42. Scully C, Hobkirk J, Dios PD: Dental endosseous implants in the medically compromised patient. J Oral Rehabil 2007;34:590-599

43. Marx RE, Johnson RP, Kline SN: Prevention of osteoradionecrosis: a randomized prospective clinical trial of hyperbaric oxygen versus penicillin. J Am Dent Assoc 1985;111:49-54

44. Taylor TD, Worthington P: Osseointegrated implant rehabilitation of the previously irradiated mandible: results of a limited trial at 3 to 7 years. J Prosthet Dent 1993;69:60-69

45. Granström G, Tjellstrom A, Branemark PI: Osseointegrated implants in irradiated bone: a case-controlled study using adjunctive hyperbaric oxygen therapy. J Oral Maxillofac Surg 1999;57:493-499

46. Schoen PJ, Raghoebar GM, Bouma J, et al: Rehabilitation of oral function in head and neck cancer patients after radiotherapy with implant-retained dentures: effects of hyperbaric oxygen therapy. Oral Oncol 2007;43:379-388
47. Schoen PJ, Raghoebar GM, Bouma J, et al: Prosthodontic rehabilitation of oral function in head-neck cancer patients with dental implants placed simultaneously during ablative tumour surgery: an assessment of treatment outcomes and quality of life. Int J Oral Maxillofac Surg 2008;37:8-16

48. De Santis D, Malchiodi L, Cucchi A, et al: Computer-assisted surgery: double surgical guides for immediate loading of implants in maxillary postextractive sites. J Craniofac Surg 2010;21:1781-1785

49. Fletcher-Stark ML, Rubenstein JE, Raigrodski AJ: The use of computer-aided manufacturing during the treatment of the edentulous mandible in an oral radiation therapy patient: clinical report. J Prosthet Dent 2011;105:154-157

50. Horowitz A, Orentlicher G, Goldsmith D: Computerized implantology for the irradiated patient. J Oral Maxillofac Surg 2009;67:619-623

51. Epstein JB, Wong FL, Stevenson-Moore P: Osteoradionecrosis: clinical experience and a proposal for classification. J Oral Maxillofac Surg 1987;45:104-110

52. Meraw SJ, Reeve CM: Dental considerations and treatment of the oncology patient receiving radiation therapy. J Am Dent Assoc 1998;129:201-205

53. De Ceulaer J, Magremanne M, van Veen A, et al: Squamous cell carcinoma recurrence around dental implants. J Oral Maxillofac Surg 2010;68:2507-2512

54. Granström G, Tjellstrom A, Branemark PI, et al: Bone-anchored reconstruction of the irradiated head and neck cancer patient. Otolaryngol Head Neck Surg 1993;108:334-343

55. Epstein JB, Lunn R, Le N, et al: Periodontal attachment loss in patients after head and neck radiation therapy. Oral Surg Oral Med Oral Pathol Oral Radiol Endod 1998;86:673-677

56. Marques MA, Dib LL: Periodontal changes in patients undergoing radiotherapy. J Periodontol 2004;75:1178-1187

57. Katsura K, Sasai K, Sato K, et al: Relationship between oral health status and development of osteoradionecrosis of the mandible: a retrospective longitudinal study. Oral Surg Oral Med Oral Pathol Oral Radiol Endod 2008;105:731738

58. Oelgiesser D, Levin L, Barak S, et al: Rehabilitation of an irradiated mandible after mandibular resection using implant/tooth-supported fixed prosthesis: a clinical report. J Prosthet Dent 2004;91:310-314

59. Smolka K, Kraehenbuehl M, Eggensperger N, et al: Fibula free flap reconstruction of the mandible in cancer patients: evaluation of a combined surgical and prosthodontic treatment concept. Oral Oncol 2008;44:571-581

60. Patel Y, Bahlhorn H, Zafar S, et al: Survey of Michigan dentists and radiation oncologists on oral care of patients undergoing head and neck radiation therapy. J Mich Dent Assoc 2012;94:34-45

61. Cuesta-Gil M, Ochandiano Caicoya S, Riba-Garcia F, et al: Oral rehabilitation with osseointegrated implants in oncologic patients. J Oral Maxillofac Surg 2009;67:2485-2496.

62. Salinas TJ, Desa VP, Katsnelson A, et al: Clinical evaluation of implants in radiated fibula flaps. J Oral Maxillofac Surg 2010;68:524-529 